\title{
NECESSIDADE DE ORIENTAÇÃO DOS TUTORES SOBRE POSSE RESPONSÁ VEL CANINA
}

\author{
NECESSITY GUIDANCE OF TUTORS OF CANINE RESPONSIBLE OWNERSHIP
}

\author{
M. F. C. PANEGOSSI ${ }^{1}$; A. E. G. W. MARQUES ${ }^{2}$; L. SILVEIRA NETO ${ }^{3}$; \\ M. G. MARQUES ${ }^{4}$; W. B. NAGATA ${ }^{5}$; K. D. S. BRESCIANI ${ }^{6}$
}

\section{RESUMO}

O objetivo dessa pesquisa foi investigar a aplicação de medidas básicas de posse responsável por tutores de cães. Para isso, foram entrevistados 50 proprietários. As questões eram referentes à procedência, detalhes ambientais e ações preventivas adotadas por eles. Apesar de alguns donos possuírem noções básicas sobre os cuidados necessários para com seus cães, existe a necessidade de se orientar a comunidade sobre posse responsável de animais de estimação, o que pode ser considerado essencial para controlar a propagação de enfermidades caninas.

PALAVRAS-CHAVE: Animais de companhia. Endoparasitose. Guarda responsável.

\section{SUMMARY}

This research's goal was to investigate the application of basic measures of responsible ownership by dog tutors. For that, 50 owners were interviewed. The questions were related to origin, environment details and preventive actions adopted by them. Although some owners had basic notions about the needed care toward their dogs, there is the need to guide the community about responsible ownership of pets, that can be considered essential to control the spread of canine diseases.

KEY-WORDS: Endoparasitosis. Pets. Responsible ownership.

\footnotetext{
${ }^{1}$ UNESP, Universidade Estadual Paulista Júlio de Mesquita Filho, Faculdade de Medicina Veterinária de Araçatuba, São Paulo. ${ }^{2}$ UNESP, Universidade Estadual Paulista Júlio de Mesquita Filho, Faculdade de Medicina Veterinária de Araçatuba, São Paulo ${ }^{3}$ UFT, Universidade Federal do Tocantins, Campus de Gurupi, Curso de Engenharia de Bioprocessos e de Biotecnologia, Tocantins.

${ }^{4}$ UNESP, Universidade Estadual Paulista Júlio de Mesquita Filho, Faculdade de Medicina Veterinária de Araçatuba, São Paulo

${ }^{5}$ UNESP, Universidade Estadual Paulista Júlio de Mesquita Filho, Faculdade de Medicina Veterinária de Araçatuba, São Paulo

${ }^{6}$ UNESP, Universidade Estadual Paulista Júlio de Mesquita Filho, Faculdade de Medicina Veterinária de Araçatuba, São Paulo
} 


\section{INTRODUÇÃO}

Cães e gatos, particularmente, exercem forte influência na rotina das pessoas, pois são considerados membros da família (SILVA et al., 2009). Nos dias de hoje, os pets significam companhia, conforto emocional, otimizam a prática de esportes e colaboram na terapia assistida de algumas enfermidades (SILVANO et al., 2010).

A intensa convivência com pequenos animais exige a adoção de medidas de guarda responsável, e estas quando não cumpridas, propiciam a disseminação de zoonoses, o que é extremamente preocupante em termos de saúde pública (WESTGARTH et al., 2010).

O proprietário deve proporcionar uma vida sadia em que estejam inclusas todas as necessidades psicológicas e fisiológicas do seu cão. A "World Society for the Protection of Animals" (WSPA) prioriza cuidados inseridos no contexto da posse responsável, visando à atenção especial à alimentação, higiene, companhia, exercícios e acompanhamento médico veterinário.

O objetivo dessa pesquisa foi investigar a aplicação de medidas básicas de posse responsável por tutores de cães.

\section{MATERIAL E MÉTODOS}

Este projeto foi aprovado pela Comissão de Ética no Uso de Animais (CEUA) da Faculdade de Medicina Veterinária UNESP, Campus de Araçatuba, Processo FOA n ${ }^{\circ}$ 2012-00760.

Para a presente pesquisa foram entrevistados 50 tutores de cães atendidos no Hospital Veterinário Luiz Quintiliano de Oliveira, Faculdade de Medicina Veterinária UNESP, Campus de Araçatuba, no período de Fevereiro a Julho de 2012. O questionário aplicado aos proprietários continham perguntas fechadas sobre seus respectivos animais de companhia: "Existem acima de cinco cães na casa?"; "Seu cão foi adotado no Centro de Controle de Zoonoses?"; "tem acesso a rua?"; "há um local específico para a defecação"; "você recolhe o material fecal"; "o animal é vermifugado"; "a administração de vermífugos foi recomendada por médicos veterinários"; "foi observado algum carrapato ou pulga?"; "o animal usa coleira repelente?"; "a ração é tampada ou protegida com sacos plásticos?”; "o animal ingere carne crua ou mal passada?"; "é castrado (a)?" e "a cadela faz uso de anticoncepcional?". Também, os tutores assinaram o Termo de Consentimento Livre e Esclarecido para participarem do estudo. Os resultados obtidos foram submetidos à análise estatística descritiva com o auxílio do programa computacional SAS® (Statistical Analysis System) versão 9.3.

\section{RESULTADOS E DISCUSSÃO}

Algumas medidas preventivas em relação ao controle de enfermidades são realizadas pelos tutores dos cães, mas outras precisam ser adotadas ainda. Os resultados obtidos no presente estudo podem ter diferido de outros anteriores devido à discrepância entre o número de entrevistados, sendo uma limitação quando se deseja tecer comparações.

$\mathrm{Na}$ Tabela 1, foi verificado que a maioria dos entrevistados tinha menos de cinco cães. A partir do último censo, de acordo com o CCZ (Centro de Controle de Zoonoses do Município de Araçatuba, Estado de São Paulo, em 2013, a média de cães domiciliados foi de 29.044, porém, é provável que esse número aumente, quando adicionados os caninos errantes.

Tabela 1 - Respostas dos tutores de cães sobre adoção de medidas de posse responsável.

\begin{tabular}{lcc}
\hline Questão & Sim \% & Não \% \\
\hline Acima de cinco cães na casa & 02 & 98 \\
Origem no Centro de Controle de Zoonoses (CCZ) & 02 & 98 \\
Acesso à rua & 96 & 04 \\
Local específico para defecação & 82 & 18 \\
Limpeza do material fecal & 84 & 16 \\
Desverminação & 92 & 8 \\
Vermífugo sem orientação de médicos veterinários & 76 & 24 \\
Infestação por ectoparasitos & 54 & 46 \\
Uso de coleira com repelente & 36 & 64 \\
Conservação do alimento (saco plástico ou tampa) & 38 & 62 \\
Fornecimento de carne crua ou mal passada & 30 & 70 \\
Castração & 30 & 70 \\
Uso de anticoncepcional & 02 & 98 \\
\hline
\end{tabular}

Considerando a aquisição dos animais, nota-se que somente um (2\%) foi adotado do CCZ. Com base, neste resultado, foi demonstrado preconceito por parte dos proprietários, talvez devido aos cães deste local terem origem desconhecida. Animais provenientes do CCZ têm contato com ambiente extremamente contaminado. A carga de patógenos é mais elevada em locais onde existe aglomeração de indivíduos, sendo portanto, maior a probabilidade de propagação de enfermidades parasitárias e infecciosas (BORG, 2003). A mescla de diferentes faixas etárias contribui para o aumento da intensidade de infecções, pois os filhotes apresentam o sistema imunológico imaturo (SILVA \& ARAÚJO, 2013). Ao serem adotados, esses cães carreiam parasitos para o novo lar, contaminando o ambiente de outros pets. 
Em relação ao acesso à rua, 96\% dos tutores responderam que o cão tem acesso à rua, com ou sem guia. Em outro estudo foi encontrado que $60 \%$ dos animais possuem acesso à rua com seu dono (LOSS et. al. 2012). O passeio deve ser sempre que possível monitorado, pois pode ser nesse momento que os cães contaminam as vias públicas com fezes que quando não recolhidas, tornam-se risco para a saúde humana e animal.

Dos entrevistados $82 \%$ afirmaram que seu pet possui local especifico para defecação. Diante disso, o material fecal do animal é recolhido com maior frequência, possibilitando maior higiene do local e menor risco de contaminação. Esta ação é de especial importância, quando se considera a elevada ocorrência de helmintos com potencial zoonótico em praças públicas (COELHO et al., 2011).

Nesta pesquisa, $84 \%$ dos entrevistados afirmaram recolher o material fecal de seus cães das vias públicas, enquanto que outros autores relataram esta atitude dos proprietários de animais de companhia em 59\% (LOSS et. al., 2012) e 25\% (DOMINGUES et al., 2015) do total de respostas analisadas.

Ao transitarem por estes locais, seres humanos podem ser alvos de parasitoses zoonóticas, como por exemplo, a Larva Migrans Cutânea (LMC). O grupo de maior risco são as crianças, pois ao entrarem em contato com solos de praças e parques públicos estão sujeitas a se infectarem com larvas de parasitos (MARQUES et. al., 2012). O agente mais frequente dessa enfermidade é o Ancylostoma braziliense, o qual habita o intestino dos caninos e elimina seus ovos pelas fezes (MACIAIS et al., 2013).

Em relação à administração de antihelmintícos, foi observado que os entrevistados ignoravam a necessidade da vermifugação no controle sistemático das endoparasitoses, ou ainda, medicavam o animal, sem a presença de médico veterinário $(76 \%)$. A utilização desses fármacos de maneira inadequada acarreta em resistência parasitária e também potencializa a contaminação ambiental (MARQUES et al., 2012).

A presença de ectoparasitos foi relatada por $54 \%$ dos donos destes pets. Esses vetores são responsáveis por alterações, como reações cutâneas e alérgicas, além do fato de terem papel indiscutível na manutenção de agentes patogênicos e na transmissão de enfermidades (SEIXAS et al., 2011).

Um dado preocupante é que a minoria dos proprietários não utilizavam coleira com repelente nos animais, visto que o Município de Araçatuba, SP, é endêmico para a Leishmaniose Visceral Canina. Essa zoonose crônica é causada por um protozoário, do gênero Leishmania, intracelular obrigatório do sistema fagocítico mononuclear, cuja transmissão ocorre por meio da picada de um vetor flebotomíneo (CORTES et al., 2012). O uso de coleiras impregnadas por inseticidas é preconizado como medida profilática para esta doença (PASSOS et al., 2014).

Em relação à conservação da ração a ser fornecida aos animais, tem-se que menos da metade dos entrevistados empregavam métodos como, por exemplo, sacos plásticos ou recipientes tampados para proteção do alimento. Essa medida é importante para impedir deposição de larvas por moscas ou mesmo contato com urina de roedores, uma vez que a exposição do alimento pode atraí-los.

Os animais que ingerem carne crua ou mal cozida estão susceptíveis a adquirirem toxoplasmose e/ou neosporose. A primeira é uma zoonose e o agente etiológico coloniza e se multiplica no epitélio do trato entérico dos felídeos, culminando na eliminação de oocistos pelas fezes, os quais, consequentemente, contaminam o meio ambiente. Homens ou cães adquirem esta enfermidade ao ingerirem essas formas evolutivas presentes no material fecal (oocistos esporulados) e cistos presentes na carne em condições citadas (STACEY et al. 2010; GALVÃO et al., 2014).

A neosporose acomete o cão, hospedeiro definitivo e este por sua vez, pode se infectar ao consumir tecidos de hospedeiros intermediários (como ruminantes) ou alimentos contaminados com oocistos esporulados expelidos por algum canídeo (LYON, 2010; ALMERÍA \& LÓPEZ-GATIUS, 2013).

No presente estudo, foi constatado mais animais castrados em comparação a resultados obtidos anteriormente (LOSS et. al., 2012). Além de evitar o aumento de cães errantes na cidade, foi comprovado que castrar fêmeas antes da idade fértil, reduz drasticamente o desenvolvimento da neoplasia mamária canina para $0,5 \%$; após o primeiro e segundo estro esse índice aumenta para $8 \%$ e 26\%, respectivamente (FILHO et al., 2010).

A maioria dos entrevistados disse ser contra o uso de anticoncepcionais para evitar a gestação das fêmeas caninas. Esses medicamentos podem ocasionar disfunção hormonal, sendo que são fatores responsáveis por aumentar a chance de desenvolvimento de tumor de mama (ANDRADE et al., 2010).

A ausência de campanhas de conscientização sobre guarda responsável dos animais leva a quadros de procriação e consequente superpopulação (LIMA \& LUNA, 2012). A guarda consciente inclui acasalamentos programados ou castrações. $\mathrm{O}$ animal deve ser acompanhado desde o nascimento até sua morte, sendo prioridade seu bem-estar (REZENDE et al., 2012).

\section{CONCLUSÃO}

Apesar de alguns tutores possuírem noções básicas sobre os cuidados necessários para com seus cães, existe a necessidade de se orientar a comunidade sobre posse responsável de animais de estimação, o que pode ser considerado essencial para controlar a propagação de enfermidades caninas.

\section{REFERÊNCIAS}

ALMERÍA, S.; LÓPEZ-GATIUS, F. Bovine neosporosis: Clinical and practical aspects. Research in Veterinary Science, v. 95, p. 303-309, 2013.

ANDRADE F. H. E., FIGUEIRO A. F. C., BERSANO P. R. O., BISSACOT D. Z. \& ROCHA N. S. Malignant mammary tumor in female dogs: environmental contaminants. Diagnostic Pathology, v. 45, p. 01-05, 2010. 
BORG, M.A.BED occupancy and overcrowding as determinant factors in the incidence of MRSA infections within general ward settings. Journal of Hospital Infection, v.54, p.316-318, 2003.

COELHO, W. M.; AMARANTE, A. F. T.; APOLINÁRIO, J. C.; COELHO, N. M. D.; BRESCIANI, K. D. S. Occurrence of Ancylostoma in dogs, cats and public places from Andradina city, São Paulo state, Brazil. Revista do Instituto de Medicina Tropical de São Paulo, v. 53, n. 4, p. 181-4, 2011.

CORTES, S.; VAZ, Y.; NEVES, R.; MAIA, C.; CARDOSO, L.; CAMPINO, L. Riskfactors for canine leishmaniasis in anendemic Mediterranean region. Veterinary Parasitology, v. 189, n.2-4, p.189-96, 2012.

DOMINGUES, L.R.; CESAR, J.A.; FASSA, A.G.; DOMINGUES, M.R. Guarda responsável de animais de estimação na área urbana do município de Pelotas, RS, Brasil. Ciência \& Saúde Coletiva, v. 20, n.1, p. 185-192, 2015.

FILHO J. C. O., KOMMERS G. D., MASUDA E. K., MARQUES B. M. F. P. P., FIGHERA R. A., IRIGOYEN L. F., BARROS C. S. L.Estudo retrospectivo de 1.647 tumores mamários em cães. Pesquisa Veterinária Brasileira, v. 30, n. 2, p. 177-185, 2010.

GALVÃO, A. L.B.; VACONCELLOS, A. L.; NAVARRO, I. T.; BRESCIANI, K. D. S. Aspectos da toxoplasmose na clínica de pequenos animais. Semina: Ciências Agrárias, v. 35, n. 1, p. 393-410, 2014.

LIMA, A. F. M; LUNA, S. P. L. Algumas causas e consequências da superpopulação canina e felina: acaso ou descaso?. Revista de Educação Continuada em Medicina Veterinária e Zootecnia do CRMV-SP / Journal of Continuing Education in Animal Science of CRMV-SP, São Paulo, v. 10, n. 1, p. 32-38, 2012.

LOSS L. D.; MUSSI J. M. S.;MELLO I. N. K.;LEÃO M. S.; FRANQUE M. P. Responsible pet ownership and dog owners conduct in the county of Alegre -ES. Acta Veterinaria Brasilica, v.6, n.2, p.105-111, 2012.

LYON, C. Update on the Diagnosis and management of Neospora caninum infections in dogs. Topical Review: Topics in Companion Animal Medicine v. 25, n. 3, p. 170-174, 2010.

MACIAS, V. C.; CARVALHO, R.; CHAVEIRO, A.; CARDOSO, J. Larva migrans cutânea - a propósito de um caso clínico. Revista da Sociedade Portuguesa de Dermatologia e Venereologia, v. 71, n.1, p.93-96, 2013.

MARQUES, J.P.; GUIMARÃES, C.R.; VILAS BOAS, A.; CARNAÚBA, P.U.; MORAES, J. Contamination of public parks and squares from Guarulhos (São Paulo State, Brazil) by Toxocara spp. and Ancylostoma spp. Revista do Instituto de Medicina Tropical de São Paulo, v. 54, p.267 - 271, 2012.
PASSOS, S. K. P.; BICALHO, A. P. C. V.; ALVES, F. S.; COELHO, M. P. R. C.; OLIVEIRA, R. D. Avaliação da eficácia da coleira impregnada de deltametrina no controle de flebotomíneos transmissores da leishmaniose visceral canina em cães banhados regularmente. In: ANAIS $35^{\circ}$ Associação Nacional dos Clínicos Veterinários de Pequenos Animais, p. 380-382, 2014.

REZENDE, L. F. G., Lopes, T. V, Maia, C. A. A, Wilson Reis Teixeira ${ }^{1}$, Vargas, S. Perfil dos proprietários de cães e gatos e a prática da guarda responsável dos acadêmicos CEULJI-ULBRA. Archives of Veterinary Science, v. 17, p.34-36, resumo 012, 2012. Suplemento.

SAS Institute Inc. The SAS System, release 9.3. SAS Institute Inc., Cary:NC, 2014.

SEIXAS, R.; ALHO, A. M.; GUERRA, D.; CARVALHO, L. M. Doenças caninas de transmissão vectorial: uma picada com muitas consequências. Veterinary Medicine, p. 18-36, 2011.

SILVA F. A. N.; CARVALHO R. L.; KLEIN R. P.; QUESSADA A. M. Responsible ownership of dogs in Buenos Aires district (Teresina, PI). Ars Veterinária, Jaboticabal, SP, v.25, n.1, p.014-017, 2009.

SILVA S. M. D.; ARAUJO F. A. P. Prevalence of infection by Giardia sp. in dogs in the municipality of Porto Alegre-RS, comparison between two populations: street dogs and dogs with owner in areas of social vulnerability. Journal of the Health Sciences Institute, n. 31, v.1, p.99-103, 2013.

SILVANO, D. BENDAS, A. J. L.; MIRANDA, M. G. N.; PINHÃO, R.; ALMEIDA, F. M.; LABARTHE, N. V.; PAIVA, J. P. Divulgação dos princípios da guarda responsável: uma vertente possível no trabalho de pesquisa a campo. Revista Eletrônica Novo Enfoque, v.09, n.09, p. 64-86, 2010.

STACEY A. ELMORE S. A.; JONES, J. L.; CONRAD, P. A.; PATTON, S.; LINDSAY, D. S.; DUBEY, J. P. Toxoplasma gondii: epidemiology, feline clinical aspects, and prevention. Trends in Parasitology, v.26, n.4, p.190-196, 2010.

WESTGARTH, C.; PINCHBECK, G. L.; BRADSHAW, J. W. S.; DAWSON, S.;GASKELL, R. M.; CHRISTLEY, R. M. Factors associated with dog ownership and contact with dogs in a UK community. BMC Veterinary Reasearch, London, v. 3, n. 5, 2007.

WORLD HEALTH ORGANIZATION. Technical Report Series 913. Geneva: WHO/WSPA, 2005. 